\title{
Prolonged Neuropsychiatric Symptoms in a Military Service Member Exposed to Mefloquine
}

\author{
Jeffrey Livezey ${ }^{1} \cdot$ Thomas Oliver $^{2} \cdot$ Louis Cantilena $^{2}$
}

Published online: 8 June 2016

(c) The Author(s) 2016. This article is published with open access at Springerlink.com

\begin{abstract}
A 32-year-old male developed neuropsychiatric symptoms 2 weeks after starting mefloquine $250 \mathrm{mg} /$ week for malaria prophylaxis. He continued to take the medication for the next 4 months. Initial symptoms included vivid dreams and anxiety, as well as balance problems. These symptoms persisted and progressed over the next 4 years to include vertigo, emotional lability, and poor short-term memory, which have greatly affected his personal and professional life. An extensive evaluation revealed objective evidence supporting a central vestibulopathy. These symptoms have been unresponsive to pharmacologic therapy and psychotherapy. A Naranjo assessment score of 6 was obtained for his initial symptoms, indicating a probable adverse drug reaction to mefloquine given the relationship between the clinical picture and drug exposure.
\end{abstract}

Jeffrey Livezey

Jeffrey.r.livezey.mil@mail.mil

1 Department of Clinical Pharmacology, Experimental Therapeutics, Walter Reed Army Institute of Research, 503 Robert Grant Ave, Silver Spring, MD 20910, USA

2 Division of Clinical Pharmacology and Medical Toxicology, Uniformed Services University of the Health Sciences, Bethesda, MD 20814, USA

\section{Key Points}

Melfoquine-induced neuropsychiatric symptoms can be severely life debilitating.

Given the overlapping symptoms of post-traumatic stress disorder and mefloquine toxicity, it can be challenging to distinguish between the two diagnoses.

Melfoquine toxicity can persist for several years after exposure has been discontinued, with little to no abatement in symptoms over time.

\section{Case Description}

A 32-year-old male United States military service member was referred to the Walter Reed National Military Medical Center (WRNMMC) Toxicology and Clinical Pharmacology Clinic for further evaluation of chronic neuropsychiatric symptoms. The patient was in his usual state of health until 2 weeks after the initiation of mefloquine $250 \mathrm{mg} /$ week for malaria prophylaxis at the start of a 6- month deployment to East Africa at the end of 2009. Compliance with prophylaxis was ensured by the medical corpsmen who witnessed the weekly medication administration as directed by the ship's Commanding Officer. The patient did not receive the Medication Guide or Information Wallet Card, and his initial symptoms included anxiety and vivid dreams. Within 2 months of the deployment the patient developed a sense of disequilibrium, as well as episodes of 
confusion. He continued to take mefloquine for the 4-month duration of the deployment, as prescribed. Aside from a 4-day-long febrile illness, for which he tested negative for malaria, he had no other medical problems during the deployment. He discussed the vivid dreams with his medic and was instructed to continue taking the mefloquine. He attributed the disequilibrium to being onboard a ship and felt that the heightened anxiety was a byproduct of being deployed. Neither symptom was discussed with his medic. He primarily stayed on a ship the entire deployment and did not partake of any local food or water and denies any significant environmental exposures during this time. However, during port stops he did report drinking alcohol while taking mefloquine. Four months into the deployment, the patient experienced a traumatic event (enemy gun fire). The patient reported that his symptoms remained constant after the traumatic event and did not change until he came home from deployment 2 months later.

As soon as he returned home from deployment, 6 months after starting mefloquine, the patient and his wife noted increased emotional lability, typically manifesting as anger and irritability. He also endorsed difficulty concentrating, a decreased interest in most activities, persistent short-term memory problems and word-finding difficulties. In addition, right after disembarking from his ship, he had persistent 'sea legs' that became more episodic over the course of a few months. At the time of his visit to WRNMMC, the episodes of vertigo occurred four to five times per day and lasted approximately 20-30 min. He described the sensation as "everything in front of me is moving". Closing his eyes improved these vertiginous symptoms. The patient reported knowing when symptoms were coming but denied any accompanying sweating, heart rate change, chest pain or nausea. He also reported having a constant dizziness that he described as "inside my head is spinning". This symptom worsens when changing position or when running. He routinely stumbles when walking and has fallen down the stairs once. Prior to his deployment, the patient was a marathon runner but is now unable to run due to disequilibrium and vertigo.

These symptoms have led to significant marital problems, as well as self-reported concerns over his parenting abilities. The patient has needed significant support from his surrounding staff to function at his job due to his continued poor short-term memory. At the time of his clinic visit, the vivid dreams persisted, as well as nightmares and recurrent insomnia. His nightmares are rarely related to his deployment experiences. Aside from three visits to a mental health clinic for anger-coping strategies, the patient has not sought medical attention for these symptoms over the previous 4 years.
Five months prior to his visit to WRNMMC, during a visit with a provider for a febrile illness, the patient mentioned the neuropsychiatric symptoms and was referred to the mental health clinic for further evaluation. He was initially diagnosed with anxiety disorder and was started on prazosin, which was stopped due to ineffectiveness. He was also tried on clonazepam and lorazepam, which were discontinued due to an increase in vertiginous symptoms and ineffectiveness, respectively. He underwent extensive neuropsychiatric testing; however, due to a perceived lack of effort and inability to focus, he failed the performance-based validity tests, making interpretation of the results difficult. After 3 months of psychotherapy and pharmacotherapy, his psychiatrist also diagnosed him with post-traumatic stress disorder (PTSD). This was primarily due to two episodes of heightened anxiety after being in close proximity to people believed to be from Somalia, which was one of the areas of operations from his previous deployment. At the time of this clinic visit, the patient was regularly seeing a psychiatrist and psychologist and had just started cognitive behavioral therapy. His medications included meclizine, venlafaxine, diazepam and clonidine. Aside from an improvement in his depressive symptoms, the patient reported no improvement in his recurrent balance or nightmare episodes since starting pharmacotherapy.

Review of symptoms was positive for daily parietal headaches that sometimes refer occipitally, typically last a few hours, and resolve without pharmacotherapy. The patient denied any accompanying photophobia, phonophobia, nausea, blurry vision or proceeding aura, and also reported having recurrent febrile episodes that lasted for 2-3 days, every 3-4 months, since his deployment. These episodes resolved without medical intervention. The patient denied any associated symptoms with the fever, including pharyngitis or aphthous ulcers. He has also had enlarged, painless cervical lymph nodes for the past year, and has denied any weight loss, skin infections, sore throats, and exposure to cats or other animals. He previously had an extensive negative workup for these, including a complete blood count (CBC), chest $\mathrm{x}$-ray, mononucleosis screen and malaria smear. Two neck ultrasounds, 6 months apart, suggested reactive lymphadenopathy. Additionally, antinuclear antibodies (ANA), folate, vitamin B12, brain magnetic resonance imaging (MRI) and internal auditory canal MRI were all normal.

Additional past medical history is significant for recurrent corneal abrasions of unknown etiology occurring approximately two to three times per year since 2009. The patient denied use of any other medications, dietary supplements or herbal remedies at the time the symptoms started or since. He reported no history of head trauma or mental health disorders. 
The patient denied any significant family medical history, including mental health disorders or a history of periodic fevers.

The patient has lived in three different, recently built homes since returning from deployment. His only hobby is fishing; he does eat the fish that he catches. The patient denied any work or home activities that involved chemical fumes or vapors or use of industrial chemicals. He reported heavy alcohol use upon returning from deployment as a means of coping with his behavioral changes. However, after a few months, the patient was able to self-limit his intake and now drinks socially. He denied a history of alcohol withdrawal symptoms and use of any illicit drugs or nicotine products.

On examination, the patient was a well-dressed, articulate man who responded appropriately to all questions. He did appear jittery and frequently had to check his smartphone for answers to several medical history questions. Significant positive findings on his physical examination included head and neck flushing and mildly enlarged right posterior cervical and submandibular chain lymph nodes, with one smooth, mobile, non-tender node on the cervical chain measuring approximately $1 \mathrm{~cm}$ in diameter. His neurological examination was normal except for loss of balance while getting up from a seated position and during the tandem walk.

The patient underwent evaluations by the Infectious Diseases, Otolaryngology, Audiology and Neurology services. His initial workup included negative urine and blood heavy metal screens (copper, arsenic, mercury, lead, cadmium), and illicit drug urine screen, normal inflammatory markers, and $\mathrm{CBC}$, thyroid, parathyroid, liver and kidney function labs. He also had a normal 24-h holter monitor. Additional laboratory examination revealed normal values for human chorionic gonadotropin (hCG), carcinoembryonic antigen (CEA), vasoactive intestinal polypeptide (VIP), catecholamines and $\alpha$-fetoprotein (AFP). His infectious disease workup included negative labs for malaria, histoplasmosis, blastomycosis, HIV, tuberculosis, bartonella, Epstein-Barr Virus (EBV), syphilis, Lyme disease, cytomegalovirus (CMV), babesiosis, and chronic $\mathrm{Q}$ fever, and microscopy was without evidence of parasites in his blood. He also underwent a fine needle aspiration of the enlarged cervical lymph node, and the pathology was consistent with benign lymphoid tissue. It was the opinion of both the infectious diseases and otolaryngology consultants that the lymphadenopathy was likely reactive and benign, and no clear infectious etiology could be identified to explain the febrile episodes.

Positive findings in the patient's initial laboratory evaluation included an elevated histamine level of $4.75 \mathrm{ng} /$ $\mathrm{mL}$ (normal <1.00), and low serotonin $(9 \mathrm{ng} / \mathrm{mL}$ [21-321 ng/mL]) and thiamine levels (42.6 nmol/L
[66.5-200 nmol/L]). As a result of the elevated histamine and flushing seen on examination, a subsequent workup to rule out a carcinoid tumor demonstrated normal 5-hydroxyindoleacetic acid (5-HIAA), chromogranin A, gastrin, adrenocorticotropic hormone (ACTH) and calcitonin levels. A repeat histamine level was also normal, as was an octreotide scan. Due to the elevated histamine and decreased serotonin, the patient's methylenetetrahydrofolate (MTHFR) gene was tested and showed a single C677T mutation. The patient also had a normal homocysteine level. He was started on oral thiamine replacement $(50 \mathrm{mg}$ twice daily), with no improvement in his symptoms.

Vestibular testing by audiology showed no evidence of peripheral vestibulopathy. An MRI of the internal auditory canals was unremarkable. Rotary chair testing results showed rare findings of hyperactive vestibulo-ocular reflex (VOR) gains and an abnormally low VOR phase. These findings were reported to be consistent with migraines, motion sickness or a central vestibulopathy. The patient was referred for vestibular rehabilitation therapy.

In follow-up, the patient has been medically discharged from the military. Four and a half years after initial mefloquine exposure, he is still experiencing significant short-term memory problems and is seeing a speech therapist to aid with techniques to improve his memory and assist in activities of daily living. As a result of his shortterm memory deficiency, the patient is only able to work part-time. He sleeps approximately 3-4 h per night and still regularly has vivid dreams and nightmares. He has multiple episodes of vertigo per week that force him to lie down and close his eyes. His constant dizziness has also persisted and he has reported several more falls while walking. His behavioral and psychological issues continue, with depressed mood, lack of motivation, restlessness, anxiety and emotional lability. He sees a behavioral therapist weekly and has discontinued all prescribed medications. He currently takes only vitamins and fish oil.

\section{Discussion}

Mefloquine was first discovered at the Walter Reed Army Institute of Research in the 1970s and was approved by the US FDA in 1989, under the tradename Lariam [1]. Although the original product insert alluded to the potential for neuropsychiatric side effects, it would be another 10-15 years before the full scope of toxicity, when used for malaria prophylaxis, was demonstrated in randomized control trials $[1,2]$. In 2003, the FDA mandated that all patients prescribed mefloquine also receive a patient medication guide. In 2013, the FDA approved an updated and more stringent mefloquine drug label that included a boxed warning which, along with the patient medication 
guide, included information that neurologic and psychologic side effects of mefloquine may persist long after the drug has been discontinued, and may become permanent $[3,4]$. Due to the increasing awareness of these neuropsychiatric side effects, the United States military relegated mefloquine to a third-line medication behind doxycycline and Malarone (atovaquone-proguanil) for prophylaxis in areas of chloroquine-resistant malaria, such as Afghanistan [5]. Consequently, from 2008 to 2013, the use of mefloquine in the military has decreased dramatically [6].

Mefloquine is an antimalarial that can be taken as treatment or as a prophylactic medication. Due to its long half-life, it is prescribed for once weekly administration for prophylactic use against malaria. Neuropsychiatric side effects attributed to mefloquine use range from common mild symptoms of insomnia, anxiety, mood changes, headaches, dizziness or vertigo to more severe and rare psychoses, violent behaviors, seizures, neuropathies and even suicide. Case reports describe a wide range of these and other symptoms, including symptoms of bipolar disorder, peripheral polyneuropathy, paranoid psychosis and suicide [7-11]. Rates of neuropsychiatric symptoms from prospective studies have ranged anywhere from 8 to $77 \%$ [12-15].

Known risk factors for these neuropsychiatric side effects include a history of neuropsychiatric disorders, female sex, no previous mefloquine exposure and low body mass index [16]. This patient, aside from this being his first exposure to mefloquine, had none of the purported risk factors for neuropsychiatric adverse drug effects.

Although case reports describing the mefloquine-induced neuropsychiatric side effects are well documented, the present case is unusual in that it documents symptoms potentially associated with mefloquine greater than 4 years after discontinuing the medication. Given the timing of the patient's initial symptoms relative to mefloquine exposure, the type of symptoms, and lack of any other plausible cause, a Naranjo assessment score of 6 was given to this patient's initial symptoms, indicating a probable relationship with mefloquine exposure. The true etiology of his progression of symptoms over the course of 4 years is more difficult to ascertain. While it is probable he suffered from mefloquine-induced toxicity during his deployment, the contribution of the traumatic event to his post-deployment behavioral changes, and the presence of other symptoms (i.e. nightmares and insomnia), serve to confound the diagnosis. The differential diagnosis includes persistent PTSD and mal de debarquement syndrome. The significant overlap in symptoms associated with mefloquine toxicity and PTSD obscures the distinction between these diagnoses. This patient exhibited behavioral symptoms in all four PTSD diagnostic clusters used in the Diagnostic and
Statistical Manual of Mental Disorders, Fifth Edition (DSM-V): re-experiencing, avoidance, negative cognitions and mood, and arousal [17]. However, as reported in the literature, the documented history of significant vestibular dysfunction as evidence by the abnormal findings on vestibular testing are more consistent with mefloquine toxicity rather than PTSD or somatoform disorders such as chronic subjective dizziness $[18,19]$. A previous case report of similar vertiginous symptoms after taking mefloquine for prophylaxis also had objective evidence of a central vestibulopathy [20]. An FDA investigation of their Adverse Event Reporting System (FAERS), as well as additional case reports, describe suspected mefloquine-induced neuropsychiatric symptoms persisting and changing over time, even after mefloquine has been discontinued $[21,22]$. A retrospective study examining mefloquine-related neuropsychiatric adverse events reported to a Danish national register showed 30 and $48 \%$ of subjects reported experiencing nightmares and some form of cognitive dysfunction respectively, for at least 9 months [22]. This supports the apparent finding in this case that mefloquine toxicity symptoms can evolve and extend well beyond the end of medication administration.

In addition to the overlap in symptoms, there exists some contrasting biochemical links between mefloquineinduced changes in the central nervous system (CNS) and the development of PTSD [23]. It has been postulated that three biochemical events (increase in markers of oxidative stress, chronic inflammation, and excitotoxicity) play roles in developing PTSD [23]. Mefloquine has also been shown to increase levels of oxidative stress markers in the CNS [24-26]. As a contrast, one of the proposed mechanisms behind mefloquine-induced neurotoxicity is gap junction, specifically connexin 36 , blockade. Connexin 36 forms the primary neuronal gap junction responsible for intercellular communication, including glutamate transmission [27]. Studies have shown that excessive glutamate release and receptor overactivation can lead to secondary neuronal death as well as PTSD [23, 28]. Pharmacologic blockade of connexin 36 can inhibit this over-activation and subsequent neuronal death [29]. In a mouse traumatic brain injury model, administration of a single dose of mefloquine $10 \mathrm{~min}$ after trauma showed a reduction in neuronal death compared with control mice [30]. However, mefloquine by itself has also been linked to neuronal cell death [31].

Some reports suggest that concomitant ethanol consumption with mefloquine may have additive toxicity [32]. This patient reported alcohol intake while on shore leave during the period he was taking mefloquine. Ethanol has also been shown to inhibit connexin 36 gap junctions connecting $\gamma$-aminobutyric acid (GABA) neurons [33]. Although plausible, it is still unclear whether concomitant 
use of ethanol increases the risk, or severity, of mefloquineinduced neuropsychiatric symptoms.

Another consideration in this case is the MTHFR heterozygous C677T mutation. The MTHFR enzyme is responsible for many functions, including folate metabolism and DNA methylation, in addition to reducing oxidative stress. Although some studies show a possible link between this mutation and psychological disorders, the literature is conflicting and no studies were found regarding mefloquine or PTSD and MTHFR mutations [34, 35].

It is well known that thiamine deficiency can also cause neurological signs and symptoms, both reversible and irreversible; however, after weeks of thiamine replacement, no improvement in symptomatology was observed in this patient. In addition, although symptoms can be varied, the patient did not exhibit the classic peripheral neuropathy or Wernicke's encephalopathy seen in dry beriberi. Interestingly, it has been shown in vitro that mefloquine completely inhibits thiamine transport [36]. How, if at all, the thiamine transport inhibition impacts mefloquine toxicity is unclear.

In light of the extensive negative workup and lack of reports linking mefloquine or PTSD with recurrent fevers, it is difficult to link the febrile episodes to the patient's current symptomatology, and these may represent repeated viral infections.

\section{Conclusion}

This case documents the potential long-term and varied mefloquine-induced neuropsychiatric side effects, ranging from a central vestibulopathy to significant behavioral changes and sleep disorders. Especially pertinent to the military population, it demonstrates the difficulty in distinguishing from possible mefloquine-induced toxicity versus PTSD, and raises some questions regarding possible linkages between the two diagnoses.

\begin{abstract}
Acknowledgments Written informed consent was obtained from the patient for publication of this case report. A copy of the written consent may be requested for review from the corresponding author. This material has been reviewed by the Walter Reed Army Institute of Research. There is no objection to its presentation and/or publication. The opinions or assertions contained herein are the private views of the author, and are not to be construed as official, or as reflecting the true views of the Department of the Army or the Department of Defense.
\end{abstract}

\section{Compliance with Ethical stndards}

Conflict of interest Jeffrey Livezey, Thomas Oliver and Louis Cantilena declare that they have no conflicts of interest.

Funding No financial support was received for the conduct of this study or the preparation of this article.
Open Access This article is distributed under the terms of the Creative Commons Attribution-NonCommercial 4.0 International License (http://creativecommons.org/licenses/by-nc/4.0/), which permits any noncommercial use, distribution, and reproduction in any medium, provided you give appropriate credit to the original author(s) and the source, provide a link to the Creative Commons license, and indicate if changes were made.

\section{References}

1. Croft A. A lesson learnt: the rise and fall of Lariam and Halfan. J R Soc Med. 2007;100:170-4.

2. Nevin R. Idiosyncratic quinoline central nervous system toxicity: historical insights into the chronic neurological sequelae of mefloquine. Int J Parasitol Drugs Drug Resist. 2014;4:118-25.

3. US FDA. FDA Drug Safety Communication: FDA approves label changes for antimalarial drug mefloquine hydrochloride due to risk of serious psychiatric and nerve side effects. 2013. Available at: http://www.fda.gov/Drugs/DrugSafety/ucm362227.htm. Accessed 4 May 2016.

4. Mefloquine hydrochloride [package insert]. Teva Pharmaceuticals. 2013. Available at: https://dailymed.nlm.nih.gov/dailymed/ drugInfo.cfm?setid=09716a24-d7da-42b2-af29-c03a1b6670bd. Accessed 3 May 2016.

5. Woodson J. Guidance on medications for prophylaxis of malaria. Washington, DC: Department of Defense Health Affairs; 2013.

6. Kersgard CM, Hickey PW. Adult malaria chemoprophylaxis prescribing patterns in the military health system from 2007-2011. Am J Trop Med Hyg. 2013;89(2):317-25.

7. Brumbaugh M, Price P, Fagan N, Hsieh H. Psychotic mania associated with mefloquine in a bipolar patient. South Med J. 2008;101:550-1.

8. Chester AC, Sandroni P. Case report: peripheral neuropathy and mefloquine prophylaxis. Am J Trop Med Hyg. 2011;85:1008-9.

9. Fuller SJ, Naraqi S, Gilessi G. Paranoid psychosis related to mefloquine antimalarial prophylaxis. $\mathrm{P} \quad \mathrm{N} G$ Med $\mathrm{J}$. 2002;45:219-21.

10. Tran TM, Browning J, Dell ML. Psychosis with paranoid delusions after a therapeutic dose of mefloquine: a case report. Malaria J. 2006;5:74.

11. Jousset N, Rouge-Maillart C, Turcant A, et al. Suicide by skull stab wounds. Am J Foren Med Path. 2010;31:378-81.

12. Nasveld P, Edstein M, Reid M, et al. Randomized, double-blind study of the safety, tolerability and efficacy of tafenoquine versus mefloquine for malaria prophylaxis in nonimmune subjects. Antimicrob Agents Chemother. 2010;54:792-8.

13. Schlagenhauf P, Steffen R, Lobel H, et al. Mefloquine tolerability during chemoprophylaxis: focus on adverse event assessments, stereochemistry and compliance. Trop Med Int Health. 1996;1:485-94.

14. van Riemsdijk M, Sturkenboom M, Ditters J, et al. Low body mass index is associated with an increased risk of neuropsychiatric adverse events and concentration impairment in women on mefloquine. Br J Clin Pharmacol. 2003;57:506-12.

15. Schlagenhauf P, Tschopp A, Johnson R, et al. Tolerability of malaria chemoprophylaxis in non-immune travelers to sub-Saharan Africa: multicenter, randomised, double blind, four arm study. BMJ. 2003;327:1-6.

16. Toovey S. Mefloquine neurotoxicity: a literature review. Travel Med Infect Dis. 2009;7:2-6.

17. American Psychiatric Association. Diagnostic and statistical manual of mental disorders. 5th ed. Washington DC: American Psychiatric Association; 2013. 
18. Nevin RL. Mefloquine and post traumatic stress disorder. In: Ritchie EC, Metzgar M, Wise D, Redding J, editors. Forensic and ethical issues in military behavioral health. Houston: Borden Institute; 2014. p. 275-96.

19. Staab JP. Chronic dizziness: the interface between psychiatry and neuro-otology. Curr Opin Neurol. 2006;19:41-8.

20. Nevin RL. Limbic encephalopathy and central vestibulopathy caused by mefloquine: a case report. Travel Med Infect Dis. 2012;10:144-51.

21. FDA Safety Alert for Human Medical Products. Lariam (mefloquine hydrochloride). FDA; 2003. Available at: http://www.fda. gov/Safety/MedWatch/SafetyInformation/SafetyAlertsforHuman MedicalProducts/ucm153319.htm. Accessed 4 May 2016.

22. Ringqvist A, Bech P, Glenthoj B, Petersen E. Acute and longterm psychiatric side effects of mefloquine: a follow-up on Danish adverse event reports. Travel Med Infect Dis. 2015;13:80-8.

23. Prasad KN, Bondy SC. Common biochemical defects linkage between post-traumatic stress disorders, mild traumatic brain injury (TBI) and penetrating TBI. Brain Res. 2015;1599:103-14.

24. Ding D, Qi W, Yu D, et al. Addition of exogenous $\mathrm{NAD}^{+}$prevents mefloquine induced neuroaxonal and hair cell degeneration through reduction of caspase-3-mediated apoptosis in cochlear organotypic cultures. PLoS One. 2013;8(11):e79817.

25. Milatovic D, Jenkins JW, Hood JE, et al. Mefloquine neurotoxicity is mediated by non-receptor tyrosine kinase. Neurotoxicology. 2011;32:578-85.

26. Hood JE, Jenkins JW, Milatovic D, et al. Mefloquine induces oxidative stress and neurodegeneration in primary rat cortical neurons. Neurotoxicology. 2010;31:518-23.
27. Bellurdo N, Trovato-Salinaro A, Mudo G, et al. Structure, chromosomal location, and brain expression of human Cx36 gene. J Neurosci Res. 1999;57:740-52.

28. Yi JH, Hazell AS. Excitotoxic mechanisms and the role of astrocytic glutamate transporters in traumatic brain injury. Neurochem Int. 2006;48:394-403.

29. Wang Y, Song JH, Denisova JV, et al. Neuronal gap junction coupling is regulated by glutamate and plays critical role in cell death during neuronal death. J Neurosci. 2012;32:713-25.

30. Belousov $\mathrm{AB}$, Wang $\mathrm{Y}$, Song $\mathrm{JH}$, et al. Neuronal gap junctions play a role in the secondary neuronal death following controlled cortical impact. Neurosci Lett. 2012;524:16-9.

31. Nevin RL. Epileptogenic potential of mefloquine chemoprophylaxis: a pathogenic hypothesis. Malaria J. 2009;8:188.

32. Wittes RC, Saguinar R. Adverse reaction to mefloquine associated with ethanol ingestion. Can Med Assoc J. 1995;152:515-7.

33. Steffenson SC, Bradley KD, Hansen DM, et al. The role of connexin-36 junctions in alcohol intoxication and consumption. Synapse. 2011;65:695-707.

34. El-Hadidy MA, Abdeen HM, El-Aziz SM, Al-Harrass M. MTHFR gene polymorphism and age of onset of schizophrenia and bipolar disorder. Biomed Res Int. 2014;2014:318483.

35. Lok A, Bockting CH, Koeter MW, et al. Interaction between the MTHFR C677T polymorphism and traumatic childhood events predicts depression. Transl Psychiatry. 2013;3:e288.

36. Huang Z, Srinivasan S, Zhang J, et al. Discovering thiamine transporters as targets of chloroquine using a novel functional genomics strategy. PLoS Genet. 2012;8(11):e1003083. 